\title{
THE NORM OF A HERMITIAN ELEMENT IN A BANACH ALGEBRA
}

\section{A. M. SINCLAIR}

ABSTRACT. We prove that the norm of a hermitian element in a Banach algebra is equal to the spectral radius of the element.

An element $h$ in a complex Banach algebra with identity (of norm 1 ) is said to be hermitian if $\|\exp i \alpha h\|=1$ for all real $\alpha$ [6], [3, Definition 5.1]. I. Vidav uses a Phragmén-Lindelöf theorem to show that the numerical radius [3, Definition 2.1] of a hermitian element is equal to its spectral radius [6, p. 123, Hilfssatz 3], [3, Theorem 5.10]. We show that the norm of $\mathrm{h}+\beta 1$ is equal to the spectral radius of $h+\beta 1$ for $h$ a hermitian element and $\beta$ a complex number (Proposition 2 ). The proof uses a generalisation of Bernstein's theorem which gives a bound on the derivative of an entire function along the real line. F. F. Bonsall and M. J. Crabb [2] have recently given an elementary proof of our Proposition 2 when $\beta$ is zero (which is equivalent to $\beta$ real). In Lemma 5 and Proposition 6 we construct a norm on the algebra of polynomials, in one indeterminate $x$, which is maximal with respect to the property that $x$ is hermitian of norm one.

An entire function $F$ is said to be of order $R$ if

$$
R=\limsup _{\alpha \rightarrow \infty} \frac{\log \log M(\alpha)}{\log \alpha}
$$

where $M(\alpha)$ denotes $\sup \{|F(z)|:|z| \leqq \alpha\}$. An entire function of finite order $R$ is said to be of type $T$ if

$$
T=\limsup _{\alpha \rightarrow \infty} \alpha^{-R} \log M(\alpha) .
$$

If the entire function $F$ is of order less than 1 or $F$ is of order 1 and type less than or equal to $T$, we say $F$ is of exponential type $T[1$, p. 8$]$. G. Lumer and R. S. Phillips [5, p. 685, Theorem 2.3] prove the following lemma when $x$ is topologically nilpotent. Let $\nu(x)$ denote the spectral radius of an element $x$.

1. Lemma. Let $A$ be a Banach algebra with identity. For each continuous linear functional $f$ on $A$ and each $x$ in $A$, the entire function $\lambda \rightarrow f(\exp \lambda x)$ is of exponential type $\nu(x)$.

Received by the editors May 18, 1970.

AMS 1968 subject classifications. Primary 4650; Secondary 3010, 3050.

Key words and phrases. Hermitian element, Banach algebra, spectral radius. 
Proof. Since $|f(\exp \lambda x)| \leqq\|f\| \cdot \exp |\lambda| \cdot\|x\|$, we see that the order of $f(\exp \lambda x)$ is less than or equal to 1 . Suppose that the order of $f(\exp \lambda x)$ is 1 . The $n$th derivative of $f(\exp \lambda x)$ at zero is $f\left(x^{n}\right)$. Thus, by equation 2.2 .12 of $[1$, p. 11], the type of $f(\exp \lambda x)$ is equal to $\lim _{\sup _{n \rightarrow \infty}}\left|f\left(x^{n}\right)\right|^{1 / n}$, which is less than or equal to the spectral radius of $x$. This completes the proof.

Alternatively Lemma 1 may be proved using [3, Theorem 3.8].

For each $x$ in $A$ that is not topologically nilpotent there is a continuous linear functional $f$ on $A$ with $\|f\|=f(1)=1$ such that $f(\exp \lambda x)$ has order 1 and type $\nu(x)$. Let $B$ be a closed commutative subalgebra of $A$ containing $x$ and 1 , and let $\theta$ be a character on $B$ such that the modulus of $\theta(x)$ is equal to $\nu(x)$. By the Hahn-Banach theorem there is an extension $f$ of $\theta$ to $A$ of norm 1. Then $f(\exp \lambda x)=\exp \lambda \theta(x)$, which is of order 1 and type $\nu(x)$.

2. Proposition. Let $A$ be a Banach algebra with identity. Then $\|h+\beta 1\|=\nu(h+\beta 1)$ for each hermitian element $h$ and each complex number $\beta$.

Proof. Because the sum of two hermitian elements is hermitian and a real multiple of the identity is hermitian [6, p. 122, Hilfssatz 2], [3, Lemma 5.4], we have to prove $\|h+\beta 1\|=\nu(h+\beta 1)$ only when $\beta$ is imaginary. Let $\boldsymbol{\gamma}$ be a real number, and let $f$ be a continuous linear functional on $A$ of norm 1 with $f(h+i \gamma 1)=\|h+i \gamma 1\|$. Then, by Lemma $1, \lambda \rightarrow f(\exp \lambda i h)$ is an entire function of exponential type $\nu(h)$ whose modulus is bounded by 1 for all real $\lambda$. We now state a generalization of a theorem of S. Bernstein [4, Theorem 1], [1, Chapter 11]. If $F$ is an entire function of exponential type $T$ whose modulus is bounded by 1 for all real $\lambda$, then

$$
\left|F^{\prime}(\lambda)-\alpha F(\lambda)\right| \leqq\left(T^{2}+\alpha^{2}\right)^{1 / 2}
$$

for all real $\lambda$ and $\alpha$, where ' denotes differentiation with respect to $\lambda$. Although the hypotheses of [4, Theorem 1] are not stated in terms of the type of an entire function it is a routine matter to write them in this form so that (1) is a special case of [4, Theorem 1]. Alternatively, when $T$ is nonzero this inequality may be obtained from inequality 11.4.5 of $[1$, p. 214$]$ by substituting $\alpha=-T \tan \omega$ (see also $[1$, p. 211 and p. 222]).

We apply (1) with $F(\lambda)=f(\exp \lambda i h)$ and $\lambda=0$ obtaining

$$
\|h+i \gamma 1\|=|f(h)+i \gamma f(1)| \leqq|\nu(h)+i \gamma|
$$

since the derivative of $f(\exp \lambda i h)$ is $f(i h \exp \lambda i h)$. Since the spectrum 
of $h$ is contained in the real line [6, p. 122, Hilfssatz 2] and $\gamma$ is real,

$$
\nu(h+i \gamma 1)=|\nu(h)+i \lambda| .
$$

Combining (2) and (3) completes the proof.

We shall require the following corollary in Proposition 6.

3. Corollary. If $Q$ is a polynomial, with complex coefficients, whose zeros lie on the imaginary axis, and if $h$ is a hermitian element, then $\|Q(h)\|=|Q(\|h\|)|$.

PRoof. The spectrum of $h$ is contained in the real line, and so, by Proposition 2, $\|h\|$ or $-\|h\|$ is in $\sigma(h)$. Thus $\nu(h-\alpha 1)=|\|h\|-\alpha|$ for all imaginary $\alpha$. Proposition 2 now implies that $\|h-\alpha 1\|=|\|h\|-\alpha|$. We factorise $Q(h)$ into linear factors and use this result and the submultiplicativity of the norm to obtain $\|Q(h)\| \leqq|Q(\|h\|)|$. As all the zeros of $Q$ lie on the imaginary axis, $|Q(\|h\|)|=|Q(-\|h\|)|$. This and the result that $\|h\|$ or $-\|h\|$ is in $\sigma(h)$ imply that $|Q(\|h\|)| \leqq \nu(Q(h))$ $\leqq\|Q(h)\|$, which completes the proof.

Alternatively Corollary 3 may be proved directly from Lemma 1 by using Theorems 11.7.7, 7.8.3, and 11.7.2 of [1].

4. Definition. Let $\boldsymbol{C}(x)$ be the algebra of all polynomials in $x$ with complex coefficients, and let $L$ be the set of all constants, and all polynomials whose zeros lie on the imaginary axis in the complex plane. Then every polynomial $P$ in $C(x)$ is the sum of a finite number of polynomials in $L$. Let $\alpha$ be positive real number. We define $\|\cdot\|_{0}$ on $\boldsymbol{C}(x)$ by

and $\|\cdot\|_{\infty}$ on $\boldsymbol{C}(x)$ by

$$
\|P\|_{0}=\inf \left\{\sum_{j}\left|Q_{j}(\alpha)\right|: P=\sum_{j} Q_{j}, Q_{j} \in L \text { all } j\right\},
$$

$$
\|P\|_{\infty}=\sup \{|P(\lambda)|:-\alpha \leqq \lambda \leqq \alpha\} .
$$

5. Lemma. Let $\alpha$ be a positive real number. Then $\|\cdot\|_{0}$ (and $\|\cdot\|_{\infty}$ ) is an algebra norm on $\mathrm{C}(x),\|\cdot\|_{0} \geqq\|\cdot\|_{\infty}$, and $x$ is a hermitian element in the completion of $\left(C(x),\|\cdot\|_{0}\right)$ with spectrum the interval $[-\alpha, \alpha]$.

Proof. If $Q$ is in $L$, then $\beta \rightarrow|Q(\beta)|$ is a monotonically increasing function of positive real $\beta$, as may be seen by factorising $Q$ into linear factors and noting that the zeros of $Q$ lie on the imaginary axis so that $\beta \rightarrow|\beta-\gamma|$ is a monotonically increasing function for each zero $\gamma$ of $Q$. Let $P=\sum_{j} Q_{j}$ with $Q_{j}$ in $L$, and let $-\alpha \leqq \lambda \leqq \alpha$. Then $|P(\lambda)|$ $\leqq \sum_{j}\left|Q_{j}(\lambda)\right|=\sum_{j}\left|Q_{j}(|\lambda|)\right|$ since $\lambda$ is real, since the zeros of $Q_{j}$ lie on the imaginary axis, and since $|\lambda+i \alpha|=|-\lambda+i \alpha|$ for all $\alpha$. There- 
fore $|P(\lambda)| \leqq \sum_{j}\left|Q_{j}(\alpha)\right|$, so that $\|P\|_{\infty} \leqq\|P\|_{0}$. If $\|P\|_{0}=0, P$ is zero on $[-\alpha, \alpha]$ and so $P=0$. An elementary calculation now shows that $\|\cdot\|_{0}$ is an algebra norm on $\mathbf{C}(x)$.

Let $A$ be the completion of $\boldsymbol{C}(x)$ in $\|\cdot\|_{0}$. Then, for all real $t, \exp i t x$ is the $\|\cdot\|_{0}=$ limit of $(1+i / n \cdot t x)^{n}$ as $n$ tends to infinity [3, Theorem 3.3]. Now $\left\|(1+i / n \cdot t x)^{n}\right\|_{0} \leqq\left|(1+i / n \cdot t \alpha)^{n}\right|$, so that, taking limits as $n$ tends to infinity, we obtain $\|\exp i t x\|_{0} \leqq|\exp i t \alpha|=1$. Therefore $\|\exp i t x\|_{0}=1$ for all real $t$, so that $x$ is a hermitian element in $A$.

Since $x$ is hermitian and $\|x\|_{0} \leqq \alpha$, the spectrum of $x$ in $A$ is contained in the interval $[-\alpha, \alpha]$. For each $\lambda$ in $[-\alpha, \alpha]$ the function $P \rightarrow P(\lambda)$ : $\boldsymbol{C}(x) \rightarrow \boldsymbol{C}$ is a continuous character on $\boldsymbol{C}(x)$ taking the value $\lambda$ at $x$. This shows that the spectrum of $x$ in $A$ is $[-\alpha, \alpha]$, and completes the proof.

The norm $\|\cdot\|_{0}$ given above is the maximal norm on $C(x)$ such that $x$ is hermitian with $\|x\|=\alpha$.

6. Proposition. Let $\alpha$ be a positive real number, let $A$ be a Banach algebra with identity, and let $h$ be in $A$. Then $h$ is hermitian with $\|h\| \leqq \alpha$ if, and only if $\|P(h)\| \leqq\|P\|_{0}$ for all $P$ in $\mathbf{C}(x)$.

Proof. If $h$ is hermitian with $\|h\| \leqq \alpha$, then, for each $Q$ in $L$, $\|Q(h)\|=|Q(\|h\|)| \leqq|Q(\alpha)|$ by Corollary 3 and the monotonicity of $|Q(\alpha)|$, which we proved in Lemma 5 . Thus $\|P(h)\| \leqq \sum_{j}\left|Q_{j}(\alpha)\right|$ for all $Q_{j}$ in $L$ with $P=\sum_{j} Q_{j}$, so that $\|P(h)\| \leqq\|P\|_{0}$ for all $P$ in $\mathbf{C}(x)$.

Conversely, suppose that $\|P(h)\| \leqq\|P\|_{0}$ for all $P$ in $\mathbf{C}(x)$. Then, by [3, Theorem 3.3],

$$
\begin{aligned}
\|\exp i t h\| & =\lim _{n \rightarrow \infty}\left\|(1+i / n \cdot t h)^{n}\right\| \leqq \lim _{n \rightarrow \infty}\left\|(1+i / n \cdot t x)^{n}\right\|_{0} \\
& =\|\exp i t x\|_{0}=1
\end{aligned}
$$

for all real $t$. This implies that $\|\exp i t h\|=1$ for all real $t$, and completes the proof since $\|h\| \leqq\|x\|_{0} \leqq \alpha$.

I wish to thank B. J. Vowden for a copy of his notes taken at F. F. Bonsall's lectures on normed algebras at the St. Andrew's Mathematical Colloquium in 1968. It was in these notes that I first saw the definition of a hermitian element in a Banach algebra. I am deeply grateful to J. R. Ringrose for advice and encouragement.

\section{REFERENCES}

1. R. P. Boas, Entire functions, Academic Press, New York, 1954. MR 16, 914.

2. F. F. Bonsall and M. J. Crabb, The spectral radius of a hermitian element of a Banach algebra, Bull. London Math. Soc. 2 (1970). 
3. F. F. Bonsall and J. Duncan, Numerical range, Bull. London Math. Soc. 2 (1970).

4. R. D. Duffin and A. C. Schaeffer, Some inequalities concerning entire functions of exponential type, Bull. Amer. Math. Soc. 43 (1937), 554-556.

5. G. Lumer and R. S. Phillips, Dissipative operators in a Banach space, Pacific J. Math. 11(1961), 679-698. MR 24 \#A2248.

6. I. Vidav, Eine metrische Kennzeichnung der selbstadjungierten Operatoren, Math. Z. 66(1956), 121-128. MR 18, 912.

University of the Witwatersrand, Johannesburg, South Africa 